\title{
EVALUACIÓN DE LA EMPATÍA EN POBLACIÓN INFANTIL ARGENTINA ${ }^{1}$
}

\author{
María Cristina Richaud de MinzI² \\ Consejo Nacional de Investigaciones Científicas y Técnicas, Argentina \\ (RECiBido el 05/09/2007, ACEPTADO El 03/04/2008)
}

\begin{abstract}
RESUMEN
Se ha encontrado que la empatía predice la conducta prosocial en la niñez media y se ha observado que los individuos empáticos son menos agresivos por su sensibilidad emocional y su capacidad para comprender las consecuencias negativas potenciales para él mismo y los otros que se pueden derivar de la agresión; por tanto, la empatía aparece negativamente relacionada con la conducta agresiva y positivamente relacionada con la conducta prosocial. La empatía incluye procesos cognitivos y experiencias afectivas y significa tanto una respuesta predominantemente cognitiva de comprensión acerca de cómo se siente el otro, como una comunión afectiva. Davis (1983) define la empatía como un constructo multidimensional. Su escala incluye componentes cognitivos: Toma de perspectiva y fantasía y componentes emocionales: preocupación empática y malestar personal. Presentaremos la adaptación argentina de The Interpersonal Reactivity Index (IRI) de Davis (1980) que consta de 28 ítems, con un formato de respuesta tipo Likert con cinco opciones de respuesta, puntuables de 0 a 4.

La versión española del IRI (Mestre, Frías y Samper, 2004) fue administrada a dos muestras de niños argentinos, de ambos sexos, de 9 a 12 años de edad, que concurrían a escuelas primarias de la ciudad de Buenos Aires, con $\mathrm{N}=633$ y 652, respectivamente.

Si bien el IRI de Davis conserva en las muestras argentinas su estructura básica, indicando que el modelo teórico de base se conserva, presenta diferencias en algunos de sus ítems en cuanto al significado que le otorgan los sujetos. Fundamentalmente, sería interesante explorar en profundidad la hipótesis de un proceso más del tipo de la inteligencia emocional que de una toma de perspectiva racional.
\end{abstract}

En lo que respecta a las demás diferencias, en general, se deben a formas culturales distintas en la interpretación de los ítems y probablemente a la influencia de la edad.

Palabras clave: Empatía, Cognición, Efectividad, Correlación, Estructura, Factor.

\footnotetext{
ABSTRACT

IIt was observed that empathy predicts prosocial behaviour in children and that empathic people are less aggressive due to their emotional sensitivity and capacity to understand the

1 El presente trabajo se realizó en el Centro Interdisciplinario de Investigaciones en Psicología Matemática y Experimental (CIIPME). Tte. Gral. Perón 2158, 1040 Buenos Aires, Argentina.

2 Directora del CIIPME, Investigadora Principal del CONICET. E-mail: minzi@ciudad.com.ar
} 
potential negative consequences for themselves and others that can arise from aggression. For this reason, empathy appears negatively related to aggression and positively related to prosocial behaviour. Empathy includes cognitive processes and affective experiences, and it means at the same time a predominantly cognitive answer of understanding about other person feelings and an emotional communion. Davis (1983) defines empathy as a multidimensional construct. His scale includes cognitive components: Perspective Taking and Fantasy, and emotional components: Empathic Concern and Personal Distress. We will present the Argentine adaptation of The Interpersonal Reactivity Index (IRI) of Davis (1980) that has 28 items, with a Likert answer format, with five options, counts from 0 to 4 . The IRI Spanish version (Mestre, Frias, \& Samper, 2004) was administered to two independent samples $(\mathrm{N}=633$ y 652, ) of Argentine children, both sexes, 9 to 12 years of age, that attended to primary schools of Buenos Aires city. Although Davis' IRI keeps, in Argentine samples, the same basic structure indicating that theoretical model is preserved, it presents for Argentine children meaning differences in some of the items. Mainly, it would be interesting to explore deeper the hypothesis about an emotional intelligence process more than a rational perspective taking. With respect to the remaining differences, they are generally due to different cultural manner in items interpretation, and probably to age influence.

Keywords: Empathy, Cognitive, Emotional, Correlation, Structure, Factor.

\section{INTRODUCCIÓN}

Para Eisenberg, el estudio de la conducta moral incluye la regulación emocional y la empatía, entendida como una respuesta emocional procedente de la comprensión del estado emotivo desarrollado en otra persona (Eisenberg, 2000).

La empatía incluye procesos cognitivos y experiencias afectivas y, dependiendo de los autores, se les da más importancia a unos que a otros. Para unos (Borke, 1971; Deutsch y Madle, 1975; Regan y Totten, 1975), la empatía es fundamentalmente un proceso cognitivo que consistiría en la habilidad cognitiva para reconocer e interpretar los sentimientos, pensamientos y puntos de vista de los demás. Esto facilita la comprensión de sus motivaciones, los sentimientos positivos hacia ellos y la conducta prosocial o altruista. Para otros (Feshbach, 1978; Hoffman, 1975, 1981, 1982, 1987), la empatía es fundamentalmente un proceso afectivo, que debe ser definido como la respuesta afectiva vicaria de los sentimientos de otra persona.

Para Gallo (1989) significa tanto una respuesta predominantemente cognitiva de comprensión acerca de cómo se siente el otro, como una comunión afectiva. Haynes y Avery (1979) caracterizaron la empatía como "la habilidad para reconocer y comprender las percepciones y sentimientos de otra persona y para expresar cuidadosamente esa comprensión en una respuesta de aceptación" (p. 527). Tal respuesta puede ser verbal o no verbal como también conductas prosociales, tales como compartir o proveer ayuda.

Es decir que por un lado, la empatía puede definirse cognitivamente en relación a la toma de perspectiva o la comprensión de los otros. Por ejemplo, Hogan (1969) describió la empatía como "la comprensión intelectual o imaginativa de la condición del otro sin experimentar realmente los sentimientos de esa persona" (p. 308). Por otra parte, la empatía se ha definido también como una reacción emocional o simpatía en respuesta a los sentimientos o experiencias de otros (Caruso y Meyer, 1998). Por ejemplo, Mehrabian y Epstein (1972) 
definieron la empatía como "la mayor receptividad a la experiencia emocional de otro" (p. 526). Finalmente, en el estudio de la empatía hay un enfoque integrativo que toma conjuntamente los aspectos cognitivo y emocional.

De todas maneras, la empatía es considerada una emoción e incluso forma parte de las denominadas emociones positivas, de manera que el aspecto cognitivo de la misma es en realidad más una inteligencia de carácter emocional que un verdadero razonamiento cognitivo.

En este sentido, Eisenberg (2002) define la empatía como una respuesta emocional que procede de la comprensión del estado o situación de otra persona y es similar a lo que la otra persona está sintiendo. Por lo tanto, la respuesta empática incluye la capacidad para comprender al otro y ponerse en su lugar, a partir de lo que se observa, de la información verbal o de información accesible desde la memoria (toma de perspectiva) y además la reacción afectiva de compartir su estado emocional, que puede producir tristeza, malestar o ansiedad. La empatía, así entendida, desempeñaría un papel central en la disposición prosocial de las personas (Eisenberg, 2000).

Dada su relevancia como precursora de la conducta prosocial altruista, es muy importante poder evaluar la empatía, aunque sus características predominantemente emocionales hacen difícil poder aprehenderla a través de instrumentos psicométricos verbales.

Davis (1983) considera a la empatía como un constructo multidimensional que incluye componentes cognitivos y emocionales y cree que en realidad constituye un conjunto de constructos relacionados con la sensibilidad hacia los otros.

Basándose en este modelo desarrolló el Interpersonal Reactivity Index (IRI) (Davis, 1980), que permite evaluar la disposición empática a través de cuatro factores, dos cognitivos y dos emocionales: Toma de perspectiva (PT), habilidad para comprender el punto de vista de la otra persona; Fantasía (FS), tendencia a identificarse con personajes del cine y de la literatura, es decir, evalúa la capacidad imaginativa del sujeto para ponerse en situaciones ficticias; Preocupación empática (EC), sentimientos de compasión, preocupación y cariño por otros; y Malestar personal (PD), sentimientos de ansiedad y malestar que el sujeto manifiesta al observar las experiencias negativas de los demás. Consta de 28 ítems con un formato de respuesta tipo Likert con cinco opciones de respuesta, puntuables de 0 a 4 .

Debido a que el IRI operacionaliza un constructo multidimensional que considera separadamente los aspectos cognitivo y afectivo postulados por los diferentes autores como constituyentes de la empatía, nos pareció una propuesta interesante para su evaluación.

El objetivo del presente trabajo es analizar el comportamiento psicométrico del IRI en niños argentinos de 9 a 12 años de edad, y determinar si mantiene la estructura factorial, es decir las dimensiones subyacentes a las que apunta, y la fiabilidad.

\section{MÉTODO}

La versión española del IRI (Mestre, Frías, y Samper, 2004) fue administrada a dos muestras de niños argentinos, de ambos sexos, de 9 a 12 años de edad, que concurrían a escuelas primarias de la ciudad de Buenos Aires, con $\mathrm{N}=633$ y 652 , respectivamente. 
En primer lugar, las respuestas de los niños de ambas muestras por separado fueron factorizadas por el método de componentes principales con rotación oblimin $($ delta $=0$ ), que es el utilizado por Davis. Luego se llevaron a cabo otros dos análisis factoriales utilizando ambas muestras conjuntamente $(\mathrm{N}=1285)$, pero separando varones $(\mathrm{N}=627)$ y mujeres (658), tal cual lo hizo el autor original, quien suponía que no había diferencias por edad, pero sí por sexo.

Se obtuvo los alpha de Cronbach por factor, para determinar la fiabilidad como consistencia interna.

Finalmente, se calculó las intercorrelaciones entre las subescalas resultantes de los análisis factoriales.

\section{RESULTADOS}

Los dos primeros análisis factoriales fueron realizados sobre dos muestras independientes de niños argentinos (tomadas en diferentes años, de distintas escuelas), para asegurarnos de que las posibles diferencias en las estructuras factoriales argentina y estadounidense no se debieran a errores de muestreo provenientes de una muestra particular. El KMO resultó de .75 (muestra 2005) y .79 (muestra 2006), indicando en ambos casos que la matriz de correlación era factorizable. En los dos casos se obtuvo cuatro factores, de acuerdo a la pendiente de Cattell y coincidiendo con el número de factores hallado por Davis.

Como puede observarse en la Tabla 1, las estructuras factoriales del IRI halladas en las dos muestras argentinas son altamente similares, aunque algo diferentes a la hallada por Davis. En el factor 1, que se ha denominado Toma de perspectiva, de los 7 ítems que definen el factor, cinco $(21,11,8,9$, y 28) coinciden con los ítems que Davis considera de toma de perspectiva y 2 (9 y 25) no. En el factor 2, Preocupación empática, de los seis ítems que definen el factor, 3 coinciden con Davis y 3 no. En el factor 3, Fantasía, de los cuatro ítems que definen el factor, los cuatro coinciden con los propuestos por Davis. Finalmente, en el Factor 4, Distrés personal, de los siete ítems que definen el factor, cuatro coinciden con la definición de Davis y tres no.

En la Tabla N. ${ }^{\circ}$ 2, aparecen los resultados de los dos análisis factoriales realizados con la muestra total $(\mathrm{N}=1285)$, separada según género y los de los análisis factoriales realizados por Davis (1980). Según puede observarse, las estructuras factoriales de varones y mujeres argentinos son altamente similares entre sí, pero presentan diferencias con las de Davis.

El primer factor, Toma de perspectiva, presenta 5 coincidencias con Davis. Sin embargo, los ítems 2. "Me preocupa y conmueve la gente con menos suerte que yo" y 9. "Tiendo a proteger a los que les toman el pelo" aparecen en este factor según la muestra argentina pero no según Davis. Por otro lado, los ítems 3. "Encuentro difícil ver las cosas desde el punto de vista de otra persona" y 15. "Si estoy seguro/a de que tengo la razón en algo, no pierdo tiempo escuchando los argumentos de los demás", que para Davis están en este factor, no lo están para la muestra argentina.

En el Factor 2, Preocupación empática, se presentan 3 coincidencias. Los ítems 20. "Me altero por las cosas que veo que ocurren alrededor" y 22. "Me describiría como una 
persona bastante sensible", que Davis considera en este factor, no aparecen pesados en la muestra argentina. Por otra parte, sí se pesan en la muestra argentina pero no en Davis, los ítems: 27. "Cuando veo a alguien que necesita urgentemente ayuda en una emergencia no sé qué hacer" (Polo negativo del factor en la muestra argentina) y 12. (-) "Me es difícil entusiasmarme con un buen libro o película".

En el Factor 3, Fantasía, se presentan 5 coincidencias, aunque dos ítems que para Davis se pesan en este factor no lo hacen en la muestra argentina: 7. (-) "No me involucro con los personajes de película u obras de teatro" y 12. (-) "Me es difícil entusiasmarme con un buen libro o película".

En el Factor 4, Distrés personal, hubo 4 coincidencias, aunque los ítems 19. (-) "Normalmente soy bastante eficaz (sé qué hacer) en situaciones difíciles"; 13. (-) "Cuando veo a alguien herido, no me asusto"; 27. "Cuando veo a alguien que necesita urgentemente ayuda en una emergencia no sé qué hacer", aparecen en este factor en Davis pero no en la muestra argentina, mientras que el 20. "Me altero por las cosas que veo que ocurren alrededor" y el 1. "Sueño, bastante a menudo, acerca de las cosas que me podrían suceder", aparecen en la muestra argentina y no en Davis.

\section{DISCUSIÓN}

El IRI de Davis, en su versión española, administrado a dos muestras de niños argentinos de 9 a 12 años de edad, de ambos sexos, ha mostrado una estructura de cuatro factores similar a la propuesta por Davis, aunque han aparecido diferencias con el original que comentaremos.

El primer factor, Toma de perspectiva, es bastante coincidente con el obtenido por Davis. Sin embargo, aparecen en el factor argentino, lo mismo que en las muestras españolas (Mestre, Frías, y Samper, 2004), los ítems 2. "Me preocupa y conmueve la gente con menos suerte que yo" y 9. "Tiendo a proteger a los que les toman el pelo", que no se pesan en el factor correspondiente de Davis. Podría hipotetizarse que los niños argentinos y españoles interpretaron estos dos ítems como ponerse afectivamente en el lugar del otro (imagino cómo me sentiría si tuviera menos suerte o imagino qué se siente cuándo a alguien le toman el pelo), posición que sería muy similar a lo expresado en los ítems en que todos acuerdan: "Antes de criticar a alguien, intento imaginar cómo me sentiría si estuviera en su lugar" o "Cuando estoy disgustado/a con alguien, intento ponerme en su lugar por un momento". Hay una diferencia muy sutil entre imaginar cómo se sentiría uno en lugar de otro sin involucrarse o involucrándose, es decir, como comentábamos antes, se trata de una comprensión emocional más que un razonamiento cognitivo. Es posible que en este proceso influya la edad, si tenemos en cuenta que la muestra argentina estaba constituida por niños de 9 a 12 años y la de Davis por alumnos ingresantes a la Universidad, es decir de alrededor de 18 años. También es interesante destacar que el ítem 20. "Me altero por las cosas que veo que ocurren alrededor" que se pesa en este factor en las muestras españolas, pero que en Davis no aparece, se pesa moderadamente (.26) pero no definitoriamente en el factor argentino, aunque nuevamente imprimiéndole al factor un carácter afectivo. En este caso, el factor debería llamarse más que Toma de 
Perspectiva, que implica un ponerse afuera, Comprensión del otro, ya que se entremezclan aspectos cognitivos y afectivos.

También en el Factor Toma de perspectiva, en la muestra argentina no aparecieron los ítems 3. "Encuentro difícil ver las cosas desde el punto de vista de otra persona" y 15. "Si estoy seguro/a de que tengo la razón en algo, no pierdo tiempo escuchando los argumentos de los demás", que para Davis están en este factor. Nótese que los dos son ítems negativos, es decir que, según Davis, es necesario invertirlos para que definan la capacidad para tomar perspectiva. Cuando se realizó el análisis factorial en la muestra argentina estos dos ítems ya se habían invertido, y es un hallazgo bastante repetido en el estudio de los tests, que un ítem al ser invertido no es comprendido exactamente como lo opuesto. Es decir que responder NO a "encuentro difícil ver las cosas ...", no quiere decir que "encuentro fácil ver las cosas...".

En el Factor 2, Preocupación empática, se presentan tres coincidencias entre la muestra argentina y la de Davis, quedando el factor argentino compuesto por los ítems 4, 14 y 18, lo mismo que en las muestras españolas (Mestre, Frías, y Samper, 2004). Por otra parte, los ítems 20. "Me altero por las cosas que veo que ocurren alrededor" y 22. "Me describiría como una persona bastante sensible", que Davis considera en este factor, no aparecen pesados en el factor de la muestra argentina. Es interesante observar que estos dos ítems definen en la muestra argentina al factor Distrés personal, lo cual podría explicarse porque "alterar" en Argentina tiene una connotación negativa casi hostil y "persona sensible" muchas veces se entiende como vulnerable. Por otra parte, sí se pesan en el Factor Preocupación empática en la muestra argentina, pero no en Davis, los ítems 27. "Cuando veo a alguien que necesita urgentemente ayuda en una emergencia no sé qué hacer", en el polo negativo del factor, 12. (-) "Me es difícil entusiasmarme con un buen libro o película", y 15. (-) "Si estoy seguro/a de que tengo la razón en algo, no pierdo tiempo escuchando los argumentos de los demás". En el caso del ítem 27 se podría hipotetizar que al estar en el polo negativo indica que cuanto más empática es la persona menos se paraliza frente a alguien que necesita ayuda, es decir más tiende a ayudar. Con respecto al ítem 12, no se refiere a la identificación con los personajes o a imaginarse estar en su lugar, como indicaría la definición de Fantasía como aspecto cognitivo de la empatía, sino al "entusiasmo" o implicación afectiva con una ficción. Finalmente, en el caso del ítem 15 , "perder tiempo" tiene una implicación más afectiva que "pensar que hay dos partes", "imaginar lo que ve el otro", "escuchar diferentes opiniones", o "intentar ponerse en el lugar del otro". Además, el ítem 15 es confuso en cuanto combina dos aspectos que deben evitarse al enunciar un ítem, como son la negación y la complejidad lógica. Si además hay que invertirlo resulta lo siguiente: (-) Si estoy seguro/a de que tengo la razón en algo, no pierdo tiempo escuchando los argumentos de los demás, que invertido indica que $\mathrm{Si}$ no estoy seguro que tengo razón pierdo tiempo escuchando... o Si estoy seguro que tengo razón pierdo tiempo escuchando..., que significan cosas distintas.

Con respecto al Factor 3, Fantasía, se presentaron cinco coincidencias. Sólo hubo dos diferencias con Davis en los ítems 7. (-) "No me involucro con los personajes de película u obras de teatro" y 12. (-) "Me es difícil entusiasmarme con un buen libro o película", ya discutidos. Con respecto al ítem 7 podría haber habido una interpretación equivocada del término involucrar. El comportamiento del ítem 1 "Sueño bastante a menudo acerca 
de las cosas que me podrían suceder" es interesante para analizar. En la muestra argentina se pesó en el Factor Fantasía al igual que en la muestra de Davis y con valores bastante similares (Argentina: .21; .28, Davis: .34, .29), pero además se pesó en el Factor Distrés personal, lo que indica que fue interpretado de dos maneras: soñar con el futuro, o soñar acerca de las cosas malas que podrían suceder, como pesadilla.

En el Factor 4, Distrés personal, hubo cuatro coincidencias, aunque los ítems 19. (-) "Normalmente soy bastante eficaz (sé qué hacer) en situaciones difíciles"; 13. (-) "Cuando veo a alguien herido, no me asusto"; 27 . "Cuando veo a alguien que necesita urgentemente ayuda en una emergencia no sé qué hacer", aparecen en este factor en Davis pero no en la muestra argentina, mientras que el 20. "Me altero por las cosas que veo que ocurren alrededor" y el 1. "Sueño, bastante a menudo, acerca de las cosas que me podrían suceder", aparecen en la muestra argentina y no en Davis. La interpretación de los ítems 27, 20 y 1 por parte de los niños argentinos ya fue comentada. Con respecto a los ítems 19 y 13 , ambos invertidos en la muestra argentina, aparecen en el polo negativo del factor Toma de perspectiva de la muestra argentina, indicando que a mayor capacidad para tomar distancia de la situación mayor capacidad para actuar frente a la crisis.

Cuando se analizaron las intercorrelaciones entre las subescalas del IRI (Tablas 3 y 4), se encontró que en las muestras argentinas, al igual que en las españolas (Mestre, Frías, y Samper, 2004), aparece una correlación significativa entre Fantasía y Toma de perspectiva, lo que acordaría con el modelo propuesto, ya que ambas tendrían que ver con el aspecto cognitivo de la empatía. Para Davis, en cambio, la correlación encontrada es modesta.

En el caso de la correlación entre Preocupación empática y Fantasía, es baja en las muestras argentinas, pero significativa en las muestras españolas y en Davis. En realidad, de acuerdo con el modelo, no debería haber correlación entre un componente cognitivo (Fantasía) y uno afectivo (Preocupación empática).

En el caso de Fantasía y Distrés Personal, en las muestras argentinas se da una correlación significativa tanto en mujeres, al igual que en las muestras españolas, como en los varones, a diferencia de las muestras españolas y de Davis.

Con respecto a Toma de perspectiva con Preocupación empática, la correlación es significativa en las mujeres en los tres tipos de muestra (argentina, española y Davis), pero no en los varones, sólo en la muestra argentina. Si tenemos en cuenta el modelo, no tendría que existir una correlación entre estos dos factores, pertenecientes a componentes diferentes, aunque en el caso de las muestras españolas y argentinas, es necesario recordar que propusimos que el factor Toma de perspectiva incluía elementos afectivos y de hecho algunos ítems que Davis consideraba pertenecientes a Preocupación Empática pasaron a formar, en las muestras argentinas y españolas, parte del factor Toma de Perspectiva.

Finalmente, en los varones argentinos la correlación entre Preocupación empática y Distrés personal es significativa y negativa, aunque no en las muestras españolas ni en Davis. En el caso de las mujeres, la correlación se hace muy baja, pero tanto en Argentina como en España aparece con signo negativo. Según el modelo, la preocupación empática y el distrés personal deberían estar relacionadas porque ambas son emocionales, pero negativamente ya que una defiende de la otra. 
Es importante notar que hubo una gran consistencia en las estructuras factoriales argentinas, tanto cuando se compararon las provenientes de dos muestras independientes, como las correspondientes a varones y mujeres, lo cual permite descartar que las diferencias con Davis se deban a errores específicos de esas muestras particulares.

Con respecto a la fiabilidad, se encontraron en la muestra argentina valores de consistencia interna dentro de los límites aceptables, siempre que se calcularan sobre las dimensiones indicadas por el análisis factorial realizado sobre las respuestas de dicha muestra, mientras que si se consideraban según la estructura factorial propuesta por Davis los valores disminuían notablemente.

\section{CONCLUSIONES}

En síntesis, la Escala de Empatía de Davis, si bien conserva en la muestra argentina su estructura básica, indicando que el modelo multidimensional propuesto por Davis se conserva, presenta diferencias en algunos de sus ítems, principalmente en cuanto al significado que le otorgan los sujetos. Fundamentalmente, en el caso del factor 1, sería interesante explorar más en profundidad la hipótesis de la existencia de un proceso más del tipo de la inteligencia emocional que de una toma de perspectiva racional. Esta diferencia se manifiesta en la operacionalización del mismo a través de comportamientos donde se entrelazan ambos procesos. También sería importante estudiar cuáles son los procesos que realmente están involucrados en la empatía, ya que las correlaciones entre las subescalas dejan dudas acerca de si las indicadas por Davis como cognitivas o afectivas lo son efectivamente, o fundamentalmente, como se comentó recién, se trata de procesos específicos de la empatía que combinan ambos aspectos. En lo que respecta a las demás diferencias, en general se deben a formas culturales distintas en la interpretación de los ítems y probablemente a la influencia de la edad, aunque según Davis tales diferencias no existirían.

\section{REFERENCIAS BIBLIOGRÁFICAS}

1. Borke, H. (1971). "Interpersonal perception of young children: Egocentrism or empathy?" Developmental Psychology, vol. 5, 263-269.

2. Caruso, D.R. \& Mayer, J.D. (1998). A Measure of Emotional Empathy for Adolescents and Adults. Manuscrito no publicado. En:www.haworthpress.com/store/E-Text/ (página visitada el 26/05/)

3. Davis, M. H. (1980). "A multidimensional approach to individual differences in empathy”. JSAS Catalog of Selected Documents in Psychology 10, 85.

4. Davis, M. H. (1983). "Measuring Individual Differences in Empathy: Evidence for a Multidimensional Approach". Journal of Personality and Social Psychology 44 (1), 113-126.

5. Deutsch, F. \& Madle, R.A. (1975). "Empathy: Historic and current conceptualizations, measurement, and a cognitive theoretical perspective". Human Development 18, 267287. 
5. Eisenberg, N. (2000). Emotion, regulation, and moral development. Annual Review of Psychology 51, 665-697.

6. Eisenberg, N. (2002). "Empathy-related emotional responses, altruism, and their socialization”. En R. J. Davidson \& A. Harrington (Eds.). Visions of compassion: Western scientists and Tibetan Buddhists examine human nature. London: Oxford University Press, pp. 135; 131-164.

7. Feshbach, N.D. (1978). Studies of empathic behaviour in children. New York: Academic Press.

8. Gallo, D. (1989). "Educating for empathy, reason and imagination". The Journal of Creative Behavior 23(2), 98-115.

9. Haynes, L.A., and Avery, A.W. (1979). "Training adolescents in self-disclosure and empathy skills". Journal of Community Psychology 26(6), 526-530

10. Hoffman, M.L. (1975). "Developmental synthesis of affect and cognition and its implications for altruistic motivation”. Developmental Psychology, 11, 607-622.

11. Hoffman, M.L. (1981). Is altruism part of human nature? Journal of Personality and social Psychology 40, 121-137.

12. Hoffman, M.L. (1982). "Development of prosocial motivaction: Empathy and guilt". En Eisemberg (Ed.). The development of prosocial behaviour. New York: Academic Press.

13. Hoffman, M.L. (1987). "The contribution of empathy to justice and moral judgment". En N. Eisemberg y J. Estrayer (Eds.), Empathy and its development. Cambridge: Cambridge University Press.

14. Hogan, R. (1969). "Development of an empathy scale". Journal of Consulting and Clinical Psychology, 33, 307-316.

15. Mehrabian, A. \& Epstein, N. (1972). “A measure of emotional empathy”. Journal of Personality 4, 525-543.

16. Mestre, V, Frias, M.D., \& Samper, P. (2004). "La medida de la empatía: Análisis del interpersonal reactivity index”. Psicothema, 16 (2), 255-260.

17. Regan, D.T., \& Totten, J. (1975). "Empathy and attribution: Turning observes into actors". Journal of Personality and social Psychology 32, 850-856. 


\section{ANEXOS}

Tabla $\mathbf{N}^{\circ}{ }^{\circ}$ 1. Análisis factoriales de muestras argentinas totales 2005 y 2006 (sujetos diferentes). N $2005=633 \quad$ N $2006=652$

\begin{tabular}{|c|c|c|c|c|c|c|c|c|}
\hline & \multicolumn{2}{|c|}{$\begin{array}{c}\text { Toma de } \\
\text { perspectiva }\end{array}$} & \multicolumn{2}{|c|}{$\begin{array}{l}\text { Preocupación } \\
\text { empática }\end{array}$} & \multicolumn{2}{|c|}{ Fantasía } & \multicolumn{2}{|c|}{$\begin{array}{l}\text { Distrés } \\
\text { personal }\end{array}$} \\
\hline & 2005 & 2006 & 2005 & 2006 & 2005 & 2006 & 2005 & 2006 \\
\hline $\begin{array}{l}\text { 21. Pienso que hay dos partes (diferentes puntos } \\
\text { de vista) para cada situación, e intento tenerlas } \\
\text { en cuenta. }\end{array}$ & .683 & .646 & -.029 & .165 & .120 & .017 & .089 & .054 \\
\hline $\begin{array}{l}\text { 11. Intento comprender mejor a mis amigos/as } \\
\text { imaginándome cómo ven ellos/as las cosas. }\end{array}$ & .635 & .617 & .058 & .142 & .183 & .045 & .134 & .234 \\
\hline $\begin{array}{l}\text { 8. Cuando tengo que decidir algo escucho diferentes } \\
\text { opiniones. }\end{array}$ & .612 & .587 & .071 & .162 & .053 & -.035 & .155 & .203 \\
\hline 9. Tiendo a proteger a los que les toman el pelo. & .551 & .523 & .006 & .044 & .104 & .138 & .171 & .107 \\
\hline $\begin{array}{l}\text { 2. Me preocupa y conmueve la gente con menos } \\
\text { suerte que yo. }\end{array}$ & .516 & .563 & -.116 & .090 & .220 & .158 & .364 & .240 \\
\hline $\begin{array}{l}\text { 25. Cuando estoy disgustado/a con alguien, intento } \\
\text { ponerme en su lugar por un momento. }\end{array}$ & .462 & .598 & .047 & .013 & .406 & .146 & .117 & .117 \\
\hline $\begin{array}{l}\text { 14. (-) Las desgracias (dolor) de otros no me } \\
\text { molestan mucho. }\end{array}$ & .227 & .067 & .566 & 609 & -.154 & .061 & -.128 & -.037 \\
\hline $\begin{array}{l}\text { 4. (-) No me preocupan los problemas de los } \\
\text { demás. }\end{array}$ & .248 & .091 & .535 & .502 & -.041 & -.016 & .048 & .095 \\
\hline $\begin{array}{l}\text { 15. (-)Si estoy seguro/a que tengo la razón en } \\
\text { algo, no pierdo tiempo escuchando los argumentos } \\
\text { de los demás. }\end{array}$ & -.105 & .165 & .500 & .497 & .016 & -.138 & .024 & -.019 \\
\hline $\begin{array}{l}\text { 18. (-) Cuando veo que otro chico está siendo } \\
\text { tratado injustamente, no siento ninguna compasión } \\
\text { por él/ella. }\end{array}$ & .388 & .000 & .495 & .589 & -.145 & -.004 & -.192 & .031 \\
\hline $\begin{array}{l}\text { 12. (-) Me es difícil entusiasmarme con un buen } \\
\text { libro o película. }\end{array}$ & .099 & .087 & .477 & .495 & -.008 & -.094 & -.236 & -.164 \\
\hline $\begin{array}{l}\text { 19.(-) Normalmente soy bastante eficaz (sé qué } \\
\text { hacer) en situaciones difíciles }\end{array}$ & -.370 & -.441 & .423 & .106 & -.071 & -.115 & .219 & .260 \\
\hline 13. (-) Cuando veo a alguien herido, no me asusto. & -.094 & -.247 & .336 & .384 & -.078 & -.126 & .390 & .164 \\
\hline $\begin{array}{l}\text { 27. Cuando veo a alguien que necesita urgentemente } \\
\text { ayuda en una emergencia no sé qué hacer. }\end{array}$ & -.269 & -.146 & -.308 & -.495 & .212 & .001 & .314 & .180 \\
\hline $\begin{array}{l}\text { 16. Después de ver una obra de teatro o cine me he } \\
\text { sentido como si fuera uno/a de los personajes. }\end{array}$ & .010 & .095 & -.064 & -.046 & .799 & .787 & .146 & .079 \\
\hline $\begin{array}{l}\text { 23. Cuando veo una buena película puedo muy } \\
\text { fácilmente ponerme en el lugar del/la protagonista. }\end{array}$ & .105 & .181 & -.072 & .056 & .737 & .722 & .049 & .161 \\
\hline 5. Me identifico con los personajes de una novela. & -.063 & .026 & -.111 & -.071 & 685 & .695 & .133 & .162 \\
\hline $\begin{array}{l}\text { 26. Cuando estoy leyendo una historia interesante } \\
\text { o una novela, imagino cómo me sentiría si los } \\
\text { acontecimientos de la historia me sucedieran a mí. }\end{array}$ & .286 & .318 & .089 & .105 & .636 & .590 & .218 & .249 \\
\hline
\end{tabular}

Continúa ... 
... viene

\begin{tabular}{|c|c|c|c|c|c|c|c|c|}
\hline 6. En situaciones de riesgo, tengo miedo. & .256 & .212 & -.141 & .029 & .146 & .078 & .582 & .665 \\
\hline $\begin{array}{l}\text { 10. Me siento vulnerable (no sé qué hacer) frente a } \\
\text { una situación muy emotiva. }\end{array}$ & .093 & .127 & .243 & .177 & .173 & .273 & .573 & .439 \\
\hline $\begin{array}{l}\text { 20. Me altero por las cosas que veo que ocurren } \\
\text { alrededor. }\end{array}$ & .276 & .257 & -.013 & -.084 & .270 & .126 & .495 & .568 \\
\hline $\begin{array}{l}\text { 24. Tiendo a perder el control frente a situaciones } \\
\text { difíciles. }\end{array}$ & -.042 & -.063 & -.048 & -.232 & .071 & .185 & .463 & .580 \\
\hline $\begin{array}{l}\text { 17. Cuando estoy en una situación emocionalmente } \\
\text { tensa, me asusto. }\end{array}$ & .047 & .118 & -.218 & -.002 & .334 & .194 & .447 & .661 \\
\hline $\begin{array}{l}\text { 22. Me describiría como una persona bastante } \\
\text { sensible. }\end{array}$ & .228 & .237 & .086 & .062 & .106 & .358 & .403 & .302 \\
\hline $\begin{array}{l}\text { 1. Sueño, bastante a menudo, acerca de las cosas } \\
\text { que me podrían suceder. }\end{array}$ & .191 & .153 & .284 & -.040 & .160 & .295 & .364 & .300 \\
\hline $\begin{array}{l}\text { 3. (-) Encuentro difícil ver las cosas desde el punto } \\
\text { de vista de otra persona. }\end{array}$ & -.128 & .009 & .255 & .349 & .009 & -.029 & -.198 & -.253 \\
\hline $\begin{array}{l}\text { 28. Antes de criticar a alguien, intento imaginar } \\
\text { cómo me sentiría si estuviera en su lugar. }\end{array}$ & .346 & .630 & -.027 & .068 & .326 & .146 & .139 & .027 \\
\hline $\begin{array}{l}\text { 7. (-) No me involucro con los personajes de película } \\
\text { u obras de teatro. }\end{array}$ & -.238 & -.056 & -.134 & -.086 & .102 & .133 & .054 & -.079 \\
\hline
\end{tabular}


EVALUACIÓN DE LA EMPATÍA EN POBLACIÓN INFANTIL ARGENTINA

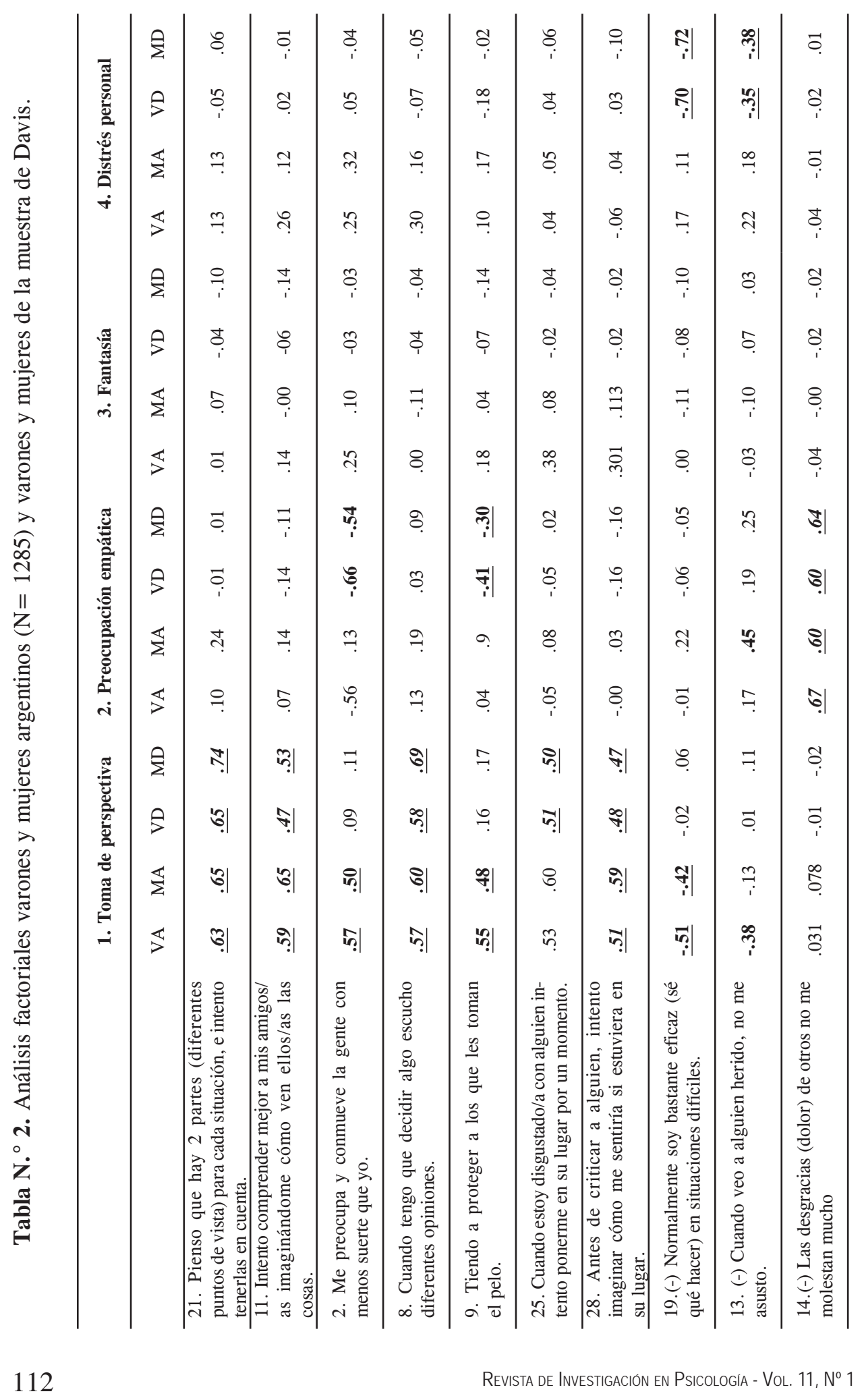




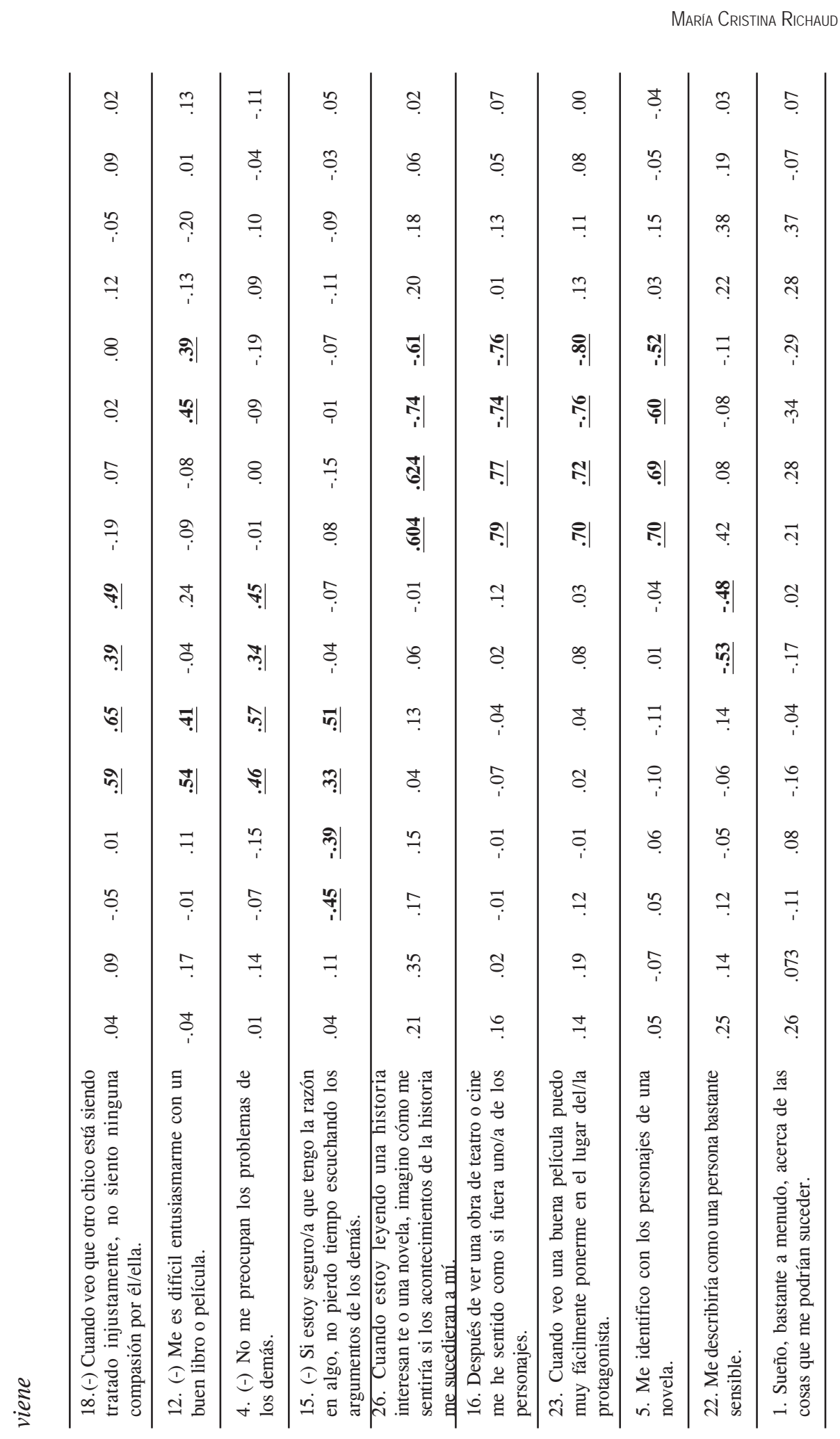

ن 
EVALUACIÓN DE LA EMPATÍA EN POBLACIÓN INFANTIL ARGENTINA

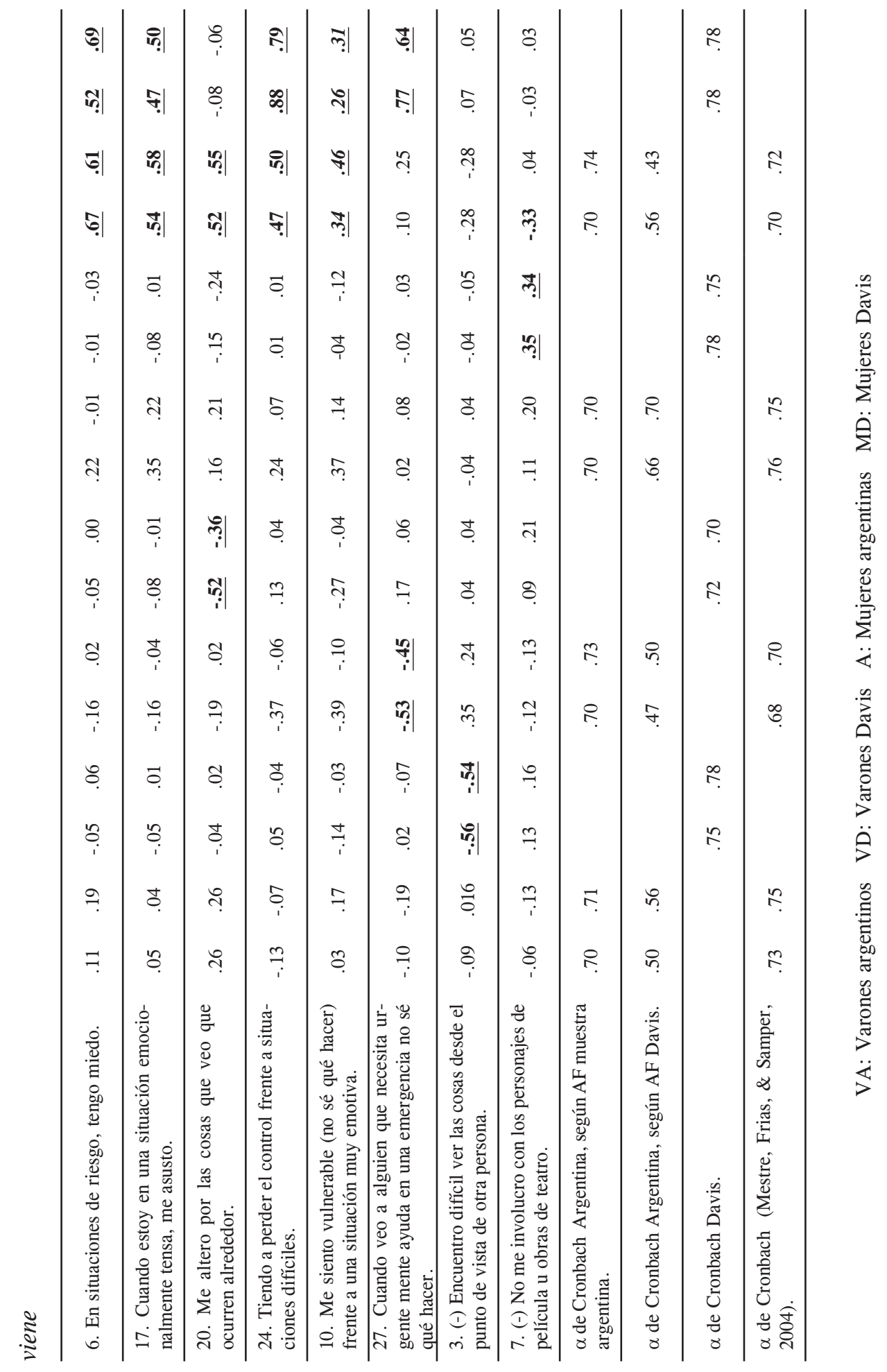


María Cristina RichaUd de M.

Tabla N. 3 . Intercorrelaciones entre las cuatro subescalas de empatía en varones.

\begin{tabular}{lccc}
\hline & $\begin{array}{c}\text { Toma de } \\
\text { perspectiva }\end{array}$ & $\begin{array}{c}\text { Preocupación } \\
\text { empática }\end{array}$ & $\begin{array}{c}\text { Distrés } \\
\text { personal }\end{array}$ \\
\hline Fantasía & $.30^{*}$ & -.07 & $.33^{*}$ \\
Argentina & .10 & .30 & .16 \\
Davis & $.17^{*}$ & .27 & .14 \\
España & & & \\
\hline Toma de perspectiva & & .06 & $.24^{*}$ \\
Argentina & .33 & -.16 \\
Davis & $.41^{*}$ & -.10 \\
España & & & \\
\hline Preocupación & & $.29 *$ \\
empática & & .11 \\
Argentina & & \\
Davis & & & \\
España & & & \\
\hline
\end{tabular}

* La correlación es significativa al nivel 0,01 (bilateral).

Tabla N. ${ }^{\circ}$ 4. Intercorrelaciones entre las cuatro subescalas de empatía en mujeres.

\begin{tabular}{lccc}
\hline & $\begin{array}{c}\text { Toma de } \\
\text { perspectiva }\end{array}$ & $\begin{array}{c}\text { Preocupación } \\
\text { empática }\end{array}$ & $\begin{array}{c}\text { Distrés } \\
\text { personal }\end{array}$ \\
\hline Fantasía & & & \\
Argentina & $.16^{*}$ & .02 & $.24^{*}$ \\
Davis & .12 & .31 & .04 \\
España & $.24^{*}$ & $.31^{*}$ & $.18^{*}$ \\
\hline Toma de perspectiva & & & \\
Argentina & & $.22^{*}$ & $.23^{*}$ \\
Davis & & .30 & -.29 \\
España & & $.32^{*}$ & $-.23^{*}$ \\
\hline
\end{tabular}

\begin{tabular}{lc} 
Preocupación & \\
empática & \\
Argentina & -.07 \\
Davis & .01 \\
España & -.10 \\
\hline
\end{tabular}

* La correlación es significativa al nivel 0,01 (bilateral). 